\title{
乌is
}

\section{Spectral density of the Dirac operator in two-flavor QCD}

\author{
Georg P. Engel, ${ }^{1}$ Leonardo Giusti, ${ }^{1}$ Stefano Lottini, ${ }^{2}$ and Rainer Sommer ${ }^{2}$ \\ ${ }^{1}$ Dipartimento di Fisica, Università di Milano-Bicocca, and INFN, Sezione di Milano-Bicocca, \\ Piazza della Scienza 3, I-20126 Milano, Italy \\ ${ }^{2}$ John von Neumann Institute for Computing (NIC), DESY, Platanenallee 6, D-15738 Zeuthen, Germany
}

(Received 19 December 2014; published 16 March 2015)

\begin{abstract}
We compute the spectral density of the (Hermitian) Dirac operator in quantum chromodynamics with two light degenerate quarks near the origin. We use CLS/ALPHA lattices generated with two flavors of $\mathrm{O}(a)$-improved Wilson fermions corresponding to pseudoscalar meson masses down to $190 \mathrm{MeV}$ and with spacings in the range $0.05-0.08 \mathrm{fm}$. Thanks to the coverage of parameter space, we can extrapolate our data to the chiral and continuum limits with confidence. The results show that the spectral density at the origin is nonzero because the low modes of the Dirac operator do condense as expected in the Banks-Casher mechanism. Within errors, the spectral density turns out to be a constant function up to eigenvalues of $\approx 80 \mathrm{MeV}$. Its value agrees with the one extracted from the Gell-Mann-Oakes-Renner relation.
\end{abstract}

DOI: 10.1103/PhysRevD.91.054505

PACS numbers: 12.38.Gc, 11.15.Ha, 12.39.Fe

\section{INTRODUCTION}

There is overwhelming evidence that the chiral symmetry group $S U\left(N_{f}\right)_{L} \times S U\left(N_{f}\right)_{R}$ of quantum chromodynamics (QCD) with a small number $N_{f}$ of light flavors breaks spontaneously to $S U\left(N_{f}\right)_{L+R}$. This progress became possible over the last decade thanks to the impressive speed-up of the numerical simulations of lattice QCD with light dynamical fermions [1-5] (for a recent compilation of results see [6]). By now it is standard practice to assume this fact and extrapolate phenomenologically interesting observables in the quark mass by applying the predictions of chiral perturbation theory (ChPT) $[7,8]$.

The distinctive signature of spontaneous symmetry breaking in QCD is the set of relations among pion masses and matrix elements which are expected to hold in the chiral limit [7]. Pions interact only if they carry momentum, and their matrix elements near the chiral limit can be expressed as known functions of two low-energy constants, the decay constant $F$ and the chiral condensate $\Sigma$. The simplest of these relations is the Gell-Mann-Oakes-Renner (GMOR) one, which equals the slope of the pion mass squared with respect to the quark mass to $2 \Sigma / F^{2}$. On the one hand, lattice simulations have become so powerful that we now have the tools to verify some of these relations with confidence. On the other hand, very little is known about the dynamical mechanism which breaks chiral symmetry. Maybe the spectrum of the Dirac operator is the simplest quantity to look at for an insight. Indeed, many years ago Banks and Casher suggested that chiral symmetry breaks if the low modes of the Dirac operator at the origin do condense and vice versa [9]. Remarkably, we now know that the spectral density [9-11] is a renormalizable quantity to which a universal meaning can be assigned [12].
The present paper is the second of two devoted to the computation of the spectral density of the Dirac operator in QCD with two flavors near the origin. ${ }^{1}$ This is achieved by extrapolating the numerical results obtained with $\mathrm{O}(a)$-improved Wilson fermions at several lattice spacings to the universal continuum limit. In the first paper, the focus was on the physics results [15], while here we report the full set of results, including the technical and numerical details of the computation. After fixing the notation and giving the parameters of the lattices simulated in Secs II and III, Secs. IV and V are devoted to two different numerical analyses of the data. Results and conclusions are given in the last section.

\section{SPECTRAL DENSITY OF THE DIRAC OPERATOR}

In a space-time box of volume $V$ with periodic boundary conditions, the spectral density of the Euclidean massless Dirac operator $D$ is defined as

$$
\rho(\lambda, m)=\frac{1}{V} \sum_{k=1}^{\infty}\left\langle\delta\left(\lambda-\lambda_{k}\right)\right\rangle
$$

where $i \lambda_{1}, i \lambda_{2}, \ldots$ are its (purely imaginary) eigenvalues ordered with their magnitude in ascending order. As usual, the bracket $\langle\ldots\rangle$ denotes the QCD expectation value and $m$ the quark mass. The spectral density is a renormalizable observable $[12,16]$. Once the free parameters in the action (coupling constant and quark masses) have been renormalized, no renormalization ambiguity is left in $\rho(\lambda, m)$. The Banks-Casher relation [9],

${ }^{1}$ Preliminary results of this work were presented in Refs. [13,14]. 


$$
\lim _{\lambda \rightarrow 0} \lim _{m \rightarrow 0} \lim _{V \rightarrow \infty} \rho(\lambda, m)=\frac{\Sigma}{\pi},
$$

links the spectral density to the chiral condensate,

$$
\Sigma=-\frac{1}{2} \lim _{m \rightarrow 0} \lim _{V \rightarrow \infty}\langle\bar{\psi} \psi\rangle,
$$

where $\psi$ is the quark doublet. It can be read in either direction. If chiral symmetry is spontaneously broken by a nonzero value of the condensate, the density of the quark modes in infinite volume does not vanish at the origin. Conversely, a nonzero density implies that the symmetry is broken.

The mode number of the Dirac operator,

$$
\nu(\Lambda, m)=V \int_{-\Lambda}^{\Lambda} \mathrm{d} \lambda \rho(\lambda, m),
$$

also corresponds to the average number of eigenmodes of the massive Hermitian operator $D^{\dagger} D+m^{2}$ with eigenvalues $\alpha \leq M^{2}=\Lambda^{2}+m^{2}$. It is a renormalization-group invariant quantity as it stands. Its (normalized) discrete derivative,

$$
\tilde{\rho}\left(\Lambda_{1}, \Lambda_{2}, m\right)=\frac{\pi}{2 V} \frac{\nu\left(\Lambda_{2}\right)-\nu\left(\Lambda_{1}\right)}{\Lambda_{2}-\Lambda_{1}},
$$

carries the same information as $\rho(\lambda, m)$, but this effective spectral density is a more convenient quantity to consider in practice on the lattice.

\section{A. Mode number on the lattice}

We discretize two-flavor QCD with the Wilson plaquette action for the gauge field and $\mathrm{O}(a)$-improved Wilson action for the doublet of mass-degenerate quarks [17,18]; see Appendix A for more details. The mode number ${ }^{2} \nu(\Lambda, m)$ is defined as the average number of eigenmodes of the massive Hermitian $\mathrm{O}(a)$-improved Wilson-Dirac operator $D_{m}^{\dagger} D_{m}$ with eigenvalues $\alpha \leq M^{2}$. In the continuum limit this definition converges to the universal one [12],

$$
\nu_{R}\left(\Lambda_{R}, m_{R}\right)=\nu(\Lambda, m),
$$

provided $m_{R}$ is defined as in Eq. (A6), and $\Lambda_{R}$ as

$$
\Lambda_{R}=\sqrt{M_{R}^{2}-m_{R}^{2}}, \quad M_{R}=Z_{\mathrm{P}}^{-1}\left(1+\bar{b}_{\mu} a m\right) M
$$

The counter term proportional to $\bar{b}_{\mu}$ ensures that at finite lattice spacing $\nu_{R}\left(M_{R}, m_{R}\right)$ is an $\mathrm{O}(a)$-improved quantity.

\footnotetext{
${ }^{2}$ We use the same notation for lattice and continuum quantities, since any ambiguity is resolved from the context. As usual, the continuum limit value of a renormalized lattice quantity, identified with the subscript $R$, is the one to be identified with its continuum counterpart.
}

This improvement coefficient has been computed in Ref. [12], and its values for the inverse couplings $\beta$ considered in this paper are given in Table III.

For Wilson fermions chiral symmetry is violated at finite lattice spacing. As a consequence, the fine details of the spectrum of the Wilson-Dirac operator near the threshold $\Lambda_{R}=0$ is not protected from large lattice effects $[16,19,20]$. While this region may be of interest for studying the peculiar details of those fermions, it is easier to extract universal information about the continuum theory far away from it. In this respect the effective spectral density in Eq. (5) is a good quantity to consider on the lattice to extract the value of the chiral condensate. ${ }^{3}$

\section{NUMERICAL SETUP}

The CLS community ${ }^{4}$ and the ALPHA Collaboration have generated the gauge configurations of the two-flavor QCD with the $\mathrm{O}(a)$-improved Wilson action by using the MP-HMC (lattices A5, B6, G8, N6 and O7) and the DD-HMC (all other lattices) algorithms as implemented in Refs. [22,23]. The primary observables that we have computed are the two-point functions of bilinear operators in Eq. (A5) and the mode number $\nu(\Lambda, m)$. The former were already computed by the ALPHA Collaboration; see Appendix B and Refs. [24,25] for more details.

\section{A. Computation of the mode number}

The stochastic computation of the mode number has been carried out as in Ref. [12]. A numerical approximation of the orthogonal projector $\mathbb{P}_{M}$ to the subspace spanned by the eigenmodes of $D_{m}^{\dagger} D_{m}$ with eigenvalues $\alpha \leq M^{2}$ is computed as

$$
\mathbb{P}_{M} \simeq h(\mathbb{X})^{4}, \quad \mathbb{X}=1-\frac{2 M_{*}^{2}}{D_{m}^{\dagger} D_{m}+M_{*}^{2}},
$$

where $M / M_{\star}=0.96334$. The function $h(x)$ is an approximation to the step function $\theta(-x)$ by a minmax polynomial of degree $n=32$ in the range $-1 \leq x \leq 1$; see Ref. [12] for more details. This choice, together with the value of $M_{\star}$ given, guarantees a systematic error well below our statistical errors. The mode number is then computed as

$$
\nu(M, m)=\left\langle\mathcal{O}_{N}\right\rangle, \quad \mathcal{O}_{N}=\frac{1}{N} \sum_{k=1}^{N}\left(\eta_{k}, \mathbb{P}_{M} \eta_{k}\right),
$$

where we have added to the theory a set of pseudofermion fields $\eta_{1}, \ldots, \eta_{N}$ with Gaussian action. In the course of a numerical simulation, one such field $(N=1)$ for each

\footnotetext{
${ }^{3}$ Once the renormalizability of the spectral density is proven, a generic finite integral of $\rho(\lambda, m)$ can be used to measure the condensate; see Ref. [21] for a different choice.

${ }^{4}$ https://wiki-zeuthen.desy.de/CLS/CLS.
} 
TABLE I. Overview of the ensembles and statistics used in this study. We give the label, the spatial extent of the lattice, $\beta=6 / g_{0}^{2}$, the hopping parameter $\kappa$ for the quark fields, the number of molecular dynamics units (MDU), the quark mass $m_{R}$ renormalized in the $\overline{\mathrm{MS}}$ scheme at $\mu=2 \mathrm{GeV}$, the pion mass $M_{\pi}$ and its decay constant $F_{\pi}$, the product $M_{\pi} L$, and the (updated) value of the lattice spacing determined as in [24] (see also [26]).

\begin{tabular}{lccccccccc}
\hline \hline id & $L / a$ & $\beta$ & $\kappa$ & MDU & $m_{R}[\mathrm{MeV}]$ & $F_{\pi}[\mathrm{MeV}]$ & $M_{\pi}[\mathrm{MeV}]$ & $M_{\pi} L$ & $a[\mathrm{fm}]$ \\
\hline A3 & 32 & 5.2 & 0.13580 & 7040 & $37.4(9)$ & $120.8(7)$ & $496(6)$ & 6.0 \\
A4 & 32 & & 0.13590 & 7920 & $22.8(6)$ & $110.7(6)$ & $386(5)$ & 4.7 \\
A5 & 32 & & 0.13594 & 1980 & $16.8(4)$ & $106.0(6)$ & $333(5)$ & 4.0 & $0.0749(8)$ \\
B6 & 48 & & 0.13597 & 1200 & $12.2(3)$ & $102.3(5)$ & $283(4)$ & 5.2 \\
E5 & 32 & 5.3 & 0.13625 & 8832 & $32.0(8)$ & $115.2(6)$ & $440(5)$ & 4.7 & $0.0652(6)$ \\
F6 & 48 & & 0.13635 & 4000 & $16.5(4)$ & $105.3(6)$ & $314(3)$ & 5.0 \\
F7 & 48 & & 0.13638 & 3600 & $12.0(3)$ & $100.9(4)$ & $268(3)$ & 4.3 & 4.1 \\
G8 & 64 & & 0.136417 & 1680 & $6.1(2)$ & $95.8(4)$ & $193(2)$ & 4.2 & $0.0483(4)$ \\
N5 & 48 & 5.5 & 0.13660 & 3840 & $34.8(8)$ & $115.1(7)$ & $443(4)$ & 5.0 \\
N6 & 48 & & 0.13667 & 7680 & $20.9(5)$ & $105.8(5)$ & $342(3)$ & 4.0 \\
O7 & 64 & & 0.13671 & 3800 & $12.9(3)$ & $101.2(4)$ & $269(3)$ & 4.2 \\
\hline \hline
\end{tabular}

gauge-field configuration is generated randomly, and the mode number is estimated in the usual way by averaging the observable $\mathcal{O}_{N}$ over the generated ensemble of fields. The mode number is an extensive quantity, and at fixed $N$ and for a given statistics, the relative statistical error of the calculated mode number is, therefore, expected to decrease like $V^{-1 / 2}$.

\section{B. Ensembles generated}

The details of the lattices are listed in Tables I and II. All of them have a size of $2 L \times L^{3}$, and the spatial dimensions are always large enough so that $M_{\pi} L \geq 4$. The three values of the coupling constant $\beta=5.2,5.3,5.5$ correspond to lattice spacings of $a=0.075,0.065,0.048 \mathrm{fm}$, respectively, which have been fixed from $F_{K}$ by supplementing the theory with a quenched "strange" quark [24]. The pion masses range from 190 to $500 \mathrm{MeV}$. To explicitly check for finite-size effects in the mode number, we have generated an additional set of lattices (D5) with the same spacing and quark mass as E5, but with a smaller lattice volume $48 \times 24^{3}$.

The autocorrelation times of the two-point functions and of the mode number are reported in Table II. For the lattice E5, we have computed $\tau_{\text {int }}(\nu)$ for three values of $a M$ corresponding to $\Lambda_{R}=30,40$ and $86 \mathrm{MeV}$, and no significative difference was observed. We thus space the measurements to give time to the mode number to decorrelate, while we bin properly the (cheaper) measurements of the two-point functions. To measure $\nu$, the number of configurations to be processed is chosen so that the statistical error of the effective spectral density receives roughly equally sized contributions from the scale and the mode number. To ensure a proper Monte Carlo sampling, a minimum of 50 configurations is processed in any case.

TABLE II. The integrated autocorrelation time $\tau_{\text {int }}$ of the pion mass and of the mode number, multiplied by the fraction of active links in the HMC $R_{\text {act }}$, is given in units of MDU. The parameters $\tau_{\text {int }}$ have a typical error of $25 \%-35 \%$. The number $n_{\text {it }}$ of MDUs skipped between two consecutive measurements of the two-point functions and of the mode number is also reported. The value of $\tau_{\exp }$ of the Markov chain given in the last column is taken from Ref. [27]. The value of $R_{\text {act }} \tau_{\text {int }}(\nu)$ for N5 is a conservative estimate from the one of E5 and a scaling proportional to $\tau_{\text {exp }}$.

\begin{tabular}{|c|c|c|c|c|c|c|}
\hline id & $R_{\text {act }}$ & $R_{\mathrm{act}} \tau_{\mathrm{int}}\left(M_{\pi}\right)$ & $R_{\mathrm{act}} n_{\mathrm{it}}\left(M_{\pi}\right)$ & $R_{\text {act }} \tau_{\text {int }}(\nu)$ & $R_{\text {act }} n_{\text {it }}(\nu)$ & $R_{\text {act }} \tau_{\exp }$ \\
\hline A3 & 0.37 & 7 & 2.96 & & 47.36 & 40 \\
\hline A4 & 0.37 & 5 & 2.96 & & 53.28 & \\
\hline A5 & 1 & 5 & 4.00 & 3 & 36.00 & \\
\hline B6 & 1 & 6 & 2.00 & & 24.00 & \\
\hline E5 & 0.37 & 9 & 5.92 & 6 & 35.52 & 55 \\
\hline F6 & 0.37 & 8 & 2.96 & & 29.60 & \\
\hline F7 & 0.37 & 7 & 2.96 & & 26.64 & \\
\hline G8 & 1 & 8 & 2.00 & & $24-48$ & \\
\hline N5 & 0.44 & 30 & 3.52 & 11 & 28.16 & 100 \\
\hline N6 & 1 & 10 & 4.00 & & 128 & \\
\hline O7 & 1 & 15 & 4.00 & & 76 & \\
\hline
\end{tabular}



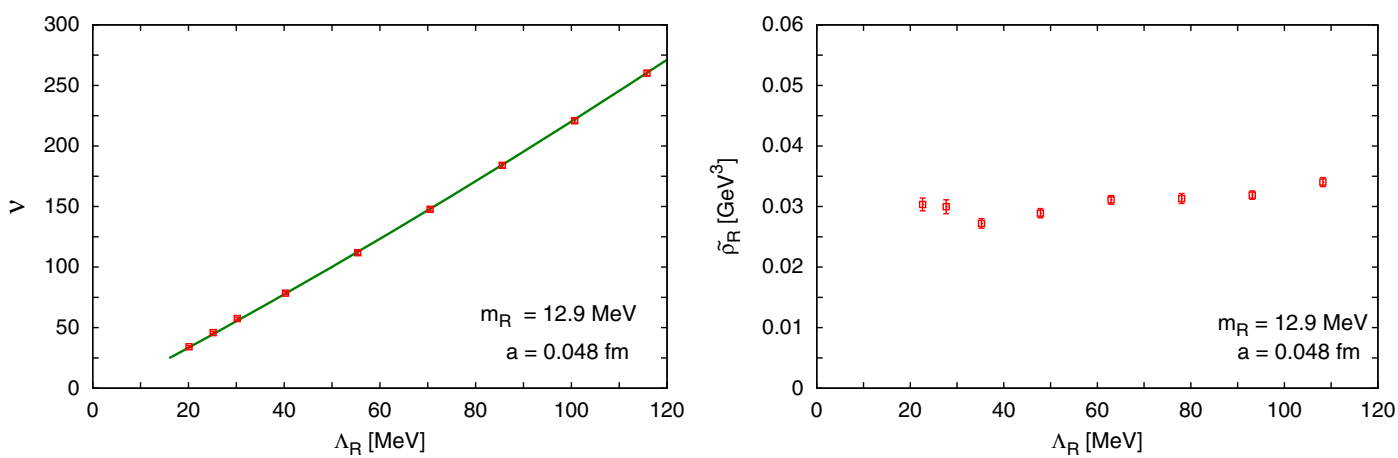

FIG. 1 (color online). Left: the mode number $\nu$ as a function of $\Lambda_{R}$ for the ensemble O7. A quadratic fit of the data gives $\nu=-9.0(13)+2.07(7) \Lambda_{R}+0.0022(4) \Lambda_{R}^{2}$. Right: the effective spectral density $\tilde{\rho}_{R}$ as defined in Eq. (5) for the same ensemble as a function of $\Lambda_{R}=\left(\Lambda_{1, R}+\Lambda_{2, R}\right) / 2$. Since we are interested in the $\Lambda_{R}$ dependence only, the errors in this plot do not include those of the lattice spacing and of $Z_{\mathrm{P}}$. The errors from $Z_{\mathrm{A}}$ and $m_{R}$ appear to be invisible in the figure.

The value of $\tau_{\exp }$ of the Markov chain, defined as in Ref. [24], is taken from [27]. It gets significantly longer towards finer lattice spacings. For the ensembles where $n_{\text {it }}<\tau_{\text {exp }}$, we estimate the contributions of the tails in the autocorrelation functions of the observables as described in Ref. [28]. When needed, we take them into account to have a more conservative error estimate.

\section{A FIRST LOOK INTO THE NUMERICAL RESULTS}

We have computed the mode number $\nu$ for nine values ${ }^{5}$ of $\Lambda_{R}$ in the range $20-120 \mathrm{MeV}$ with a statistical accuracy of a few percent on all lattices listed in Table I. Four larger values of $\Lambda_{R}$ in the range $150-500 \mathrm{MeV}$ also have been analyzed for the ensemble E5. The results are collected in Tables V-VII of Appendix D.

In Fig. 1 we show $\nu$ as a function of $\Lambda_{R}$ for the lattice O7, corresponding to the smallest reference quark mass (see below) at the smallest lattice spacing. On all other lattices an analogous qualitative behavior is observed. The mode number is a nearly linear function in $\Lambda_{R}$ up to approximatively $100-150 \mathrm{MeV}$. A clear departure from linearity is observed for $\Lambda_{R}>200 \mathrm{MeV}$ on the lattice E5. At the percent precision, however, the data show statistically significant deviations from the linear behavior already below $100 \mathrm{MeV}$. To guide the eye, a quadratic fit in $\Lambda_{R}$ is shown in Fig. 1, and the values of the coefficients are given in the caption. The bulk of $\nu$ is given by the linear term, while the constant and the quadratic term represent $\mathrm{O}(10 \%)$ corrections in the fitted range. The nearly linear behavior of the mode number is manifest on the right plot of Fig. 1, where its discrete derivative, defined as in Eq. (5) for each couple of consecutive values of $\Lambda_{R}$, is shown as a function of $\Lambda_{R}=\left(\Lambda_{1, R}+\Lambda_{2, R}\right) / 2$. Since it is not affected

\footnotetext{
${ }^{5}$ If not explicitly stated, the scheme- and scale-dependent quantities such as $\Sigma, m_{R}, \Lambda_{R}$ and $\tilde{\rho}_{R}$ are renormalized in the $\overline{\mathrm{MS}}$ scheme at $\mu=2 \mathrm{GeV}$.
}

by threshold effects, the effective spectral density $\tilde{\rho}_{R}$ is the primary observable we focus on in the next sections.

\section{A. Continuum-limit extrapolation}

In general for $\tilde{\rho}_{R}$ we observe quite a flat behavior in $\Lambda_{R}$ toward finer lattice spacings and light quark masses, similar to the one shown in Fig. 1. Because the action and the mode number are $\mathrm{O}(a)$-improved, the Symanzik effective theory analysis predicts that discretization errors start at $\mathrm{O}\left(a^{2}\right)$. In order to remove them, at every lattice spacing we match three quark mass values $\left(m_{R}=12.9,20.9,32.0 \mathrm{MeV}\right)$ by interpolating $\tilde{\rho}_{R}$ linearly in $m_{R}$ (see next section for more details). The values of $\tilde{\rho}_{R}$ show mild discretization effects at light $m_{R}$ and $\Lambda_{R}$, while they differ up to $15 \%$ per linear dimension among the three lattice spacings toward larger $\Lambda_{R}$. Within the statistical errors all data sets are compatible with a linear dependence in $a^{2}$, and we thus independently extrapolate each triplet of points to the continuum limit accordingly. We show six of those extrapolations in Fig. 2, considering the lightest and the heaviest reference quark masses for the lightest, an intermediate, and the heaviest cutoff $\Lambda_{R}$. The difference between the values of $\tilde{\rho}_{R}$ at the finest lattice spacing and the continuum-extrapolated ones is within the statistical errors for light $m_{R}$ and $\Lambda_{R}$, and it remains within few standard deviations toward larger values of $m_{R}$ and $\Lambda_{R}$. This fact makes us confident that the extrapolation removes the cutoff effects within the errors quoted.

The results for $\tilde{\rho}_{R}$ at $m_{R}=12.9 \mathrm{MeV}$ in the continuum limit are shown as a function of $\Lambda_{R}$ in the left plot of Fig. 3. A similar $\Lambda_{R}$ dependence is observed at the two other reference masses. It is worth noting that no assumption on the presence of spontaneous symmetry breaking was needed so far. These results, however, point to the fact that the spectral density of the Dirac operator in two-flavor QCD is (almost) constant in $\Lambda_{R}$ near the origin at small quark masses. This is consistent with the expectation from the Banks-Casher relation in presence of spontaneous 

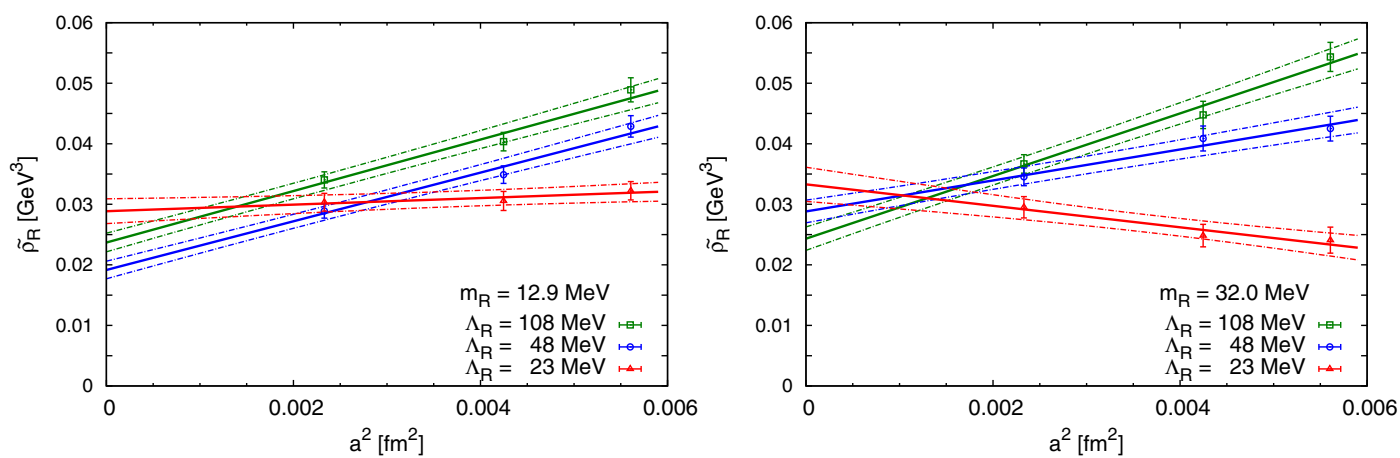

FIG. 2 (color online). Effective spectral density $\tilde{\rho}_{R}$ vs the lattice spacing squared for the lightest (left-hand side) and the heaviest reference quark mass $m_{R}$ (right-hand side), and for the lightest, an intermediate, and the heaviest cutoff $\Lambda_{R}$ in both panels. In general, the data are well described by a linear fit in $a^{2}$, which suggests that, within our statistical errors, we are in the asymptotic regime of Symanzik effective theory. As evident from the figures, there are competing (positive and negative) discretization effects, which can approximately compensate for each other in specific domains of parameter space.

symmetry breaking. In this case next-to-leading (NLO) ChPT indeed predicts

$$
\begin{aligned}
\tilde{\rho}_{R}^{\text {nlo }}= & \Sigma\left\{1+\frac{m_{R} \Sigma}{(4 \pi)^{2} F^{4}}\left[3 \bar{l}_{6}+1-\ln (2)\right.\right. \\
& \left.\left.-3 \ln \left(\frac{\Sigma m_{R}}{F^{2} \bar{\mu}^{2}}\right)+\tilde{g}_{\nu}\left(\frac{\Lambda_{1, R}}{m_{R}}, \frac{\Lambda_{2, R}}{m_{R}}\right)\right]\right\},
\end{aligned}
$$

i.e. an almost flat function in (small) $\Lambda_{R}$ at (small) finite quark masses; see Appendix $\mathrm{C}$ for unexplained notation. At fixed quark mass, the $\Lambda_{R}$ dependence of $\tilde{\rho}_{R}{ }^{\text {nlo }}$ in Eq. (10) is parameter free once the pion mass and decay constant are measured.

\section{B. Chiral limit}

The extrapolation to the chiral limit requires an assumption on how the effective spectral density $\tilde{\rho}_{R}$ behaves when $m_{R} \rightarrow 0$. In this respect it is interesting to note that the NLO function in Eq. (10) goes linearly in $m_{R}$ near the chiral limit since there are no chiral logarithms at fixed $\Lambda_{R}$; see Appendix C. A fit of the data to Eq. (10)

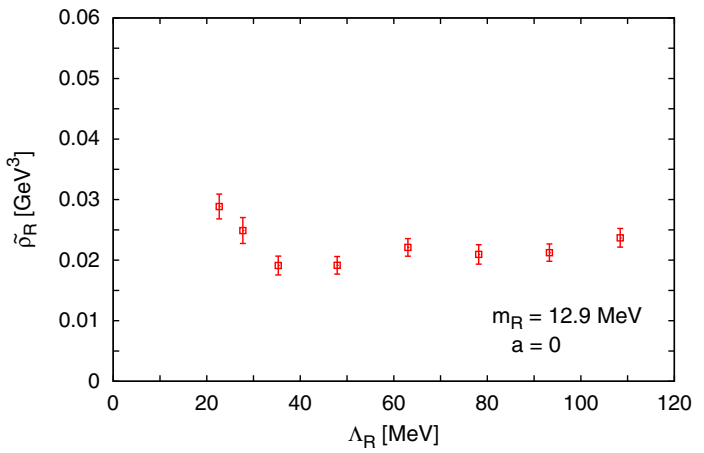

shows that the data are compatible with that NLO formula. A prediction of NLO ChPT in the two-flavor theory is that in the chiral limit $\tilde{\rho}_{R}^{\text {nlo }}=\Sigma$ also at nonzero $\Lambda_{R}$, since all NLO corrections in Eq. (10) vanish [29]. To check for this property we extrapolate $\tilde{\rho}_{R}$ with Eq. (11), which is a generalization of Eq. (10) see below, and we obtain the results shown in the right plot of Fig. 3 with a $\chi^{2} /$ dof $=$ $16.4 / 14$. Within errors the $\Lambda_{R}$ dependence is clearly compatible with a constant up to $\approx 80 \mathrm{MeV}$. Moreover the difference between the values of $\tilde{\rho}_{R}$ in the chiral limit and those at $m_{R}=12.9 \mathrm{MeV}$ is of the order of the statistical error; i.e., the extrapolation is very mild. A fit to a constant of the data gives $\Sigma^{1 / 3}=261(6) \mathrm{MeV}$.

As in any numerical computation, the chiral limit inevitably requires an extrapolation of the results with a pre-defined functional form. The distinctive feature of spontaneous symmetry breaking, however, is that the behavior of $\tilde{\rho}_{R}$ near the origin is predicted by ChPT, and its extrapolated value has to agree with the one of $M_{\pi}^{2} F_{\pi}^{2} /\left(2 m_{R}\right)$. We have thus complemented our computations with those for $m_{R}, M_{\pi}$ and $F_{\pi}$, and extrapolated the above-mentioned ratio to the chiral limit as prescribed by

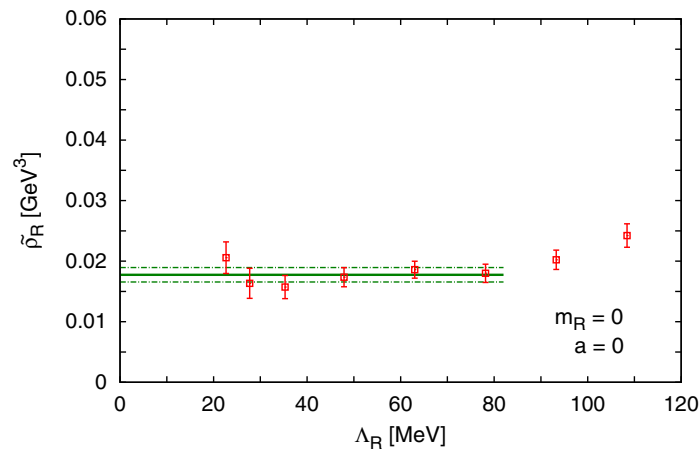

FIG. 3 (color online). Effective spectral density $\tilde{\rho}_{R}$ in the continuum limit at the smallest reference quark mass $m_{R}=12.9 \mathrm{MeV}(\mathrm{left})$, and in the chiral limit (right). Note the flat dependence on $\Lambda_{R}$ which agrees with the expectation from NLO ChPT. The results of the fit to a constant is also shown on the right plot. 

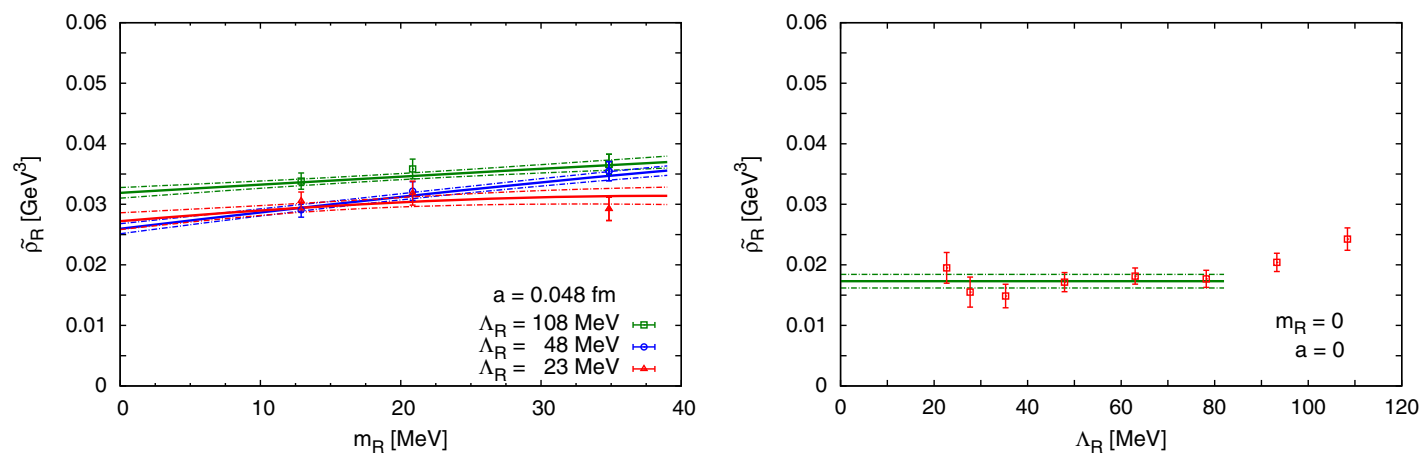

FIG. 4 (color online). Left: effective spectral density $\tilde{\rho}_{R}$ vs the quark mass $m_{R}$ for the finer lattice spacings and three cutoffs $\Lambda_{R}$ together with the combined fit to all data to Eq. (14). Right: effective spectral density $\tilde{\rho}_{R}$ vs the cutoff $\Lambda_{R}$ in the continuum and chiral limits. The squares are the results for $c_{0,0}\left(\Lambda_{R}\right)$ of the fit to the function in Eq. (14), and the plateau fit shown gives the value for the chiral condensate.

ChPT; see Appendix B and Ref. [15]. We obtain $\Sigma_{\mathrm{GMOR}}^{1 / 3}=$ 263(3)(4) MeV, where the first error is statistical and the second is systematic, in excellent agreement with the value quoted above. These results show that the spectral density at the origin has a nonzero value in the chiral limit. In the rest of this paper we assume this conclusion, and we apply standard field theory arguments to remove with confidence the (small) contributions in the raw data due to the discretization effects, the finite quark mass and finite $\Lambda_{R}$.

\section{DETAILED DISCUSSION OF NUMERICAL RESULTS}

We have analyzed the numerical results for the effective spectral density $\tilde{\rho}_{R}$ following two different fitting strategies. In the first one, the main results of which are reported in the previous section, we have extrapolated the results at fixed kinematics $\left(\Lambda_{R}, m_{R}\right)$ to the continuum limit independently. The results of this analysis call for an alternative strategy to extract the chiral condensate which uses ChPT from the starting point, i.e. based on fitting the data in all three directions $\left(\Lambda_{R}, m_{R}, a\right)$ at the same time. This procedure reduces the number of fit parameters, allows us to include all generated data in the fit, and avoids the need for an interpolation in the quark mass. It is important to stress that also in this case ChPT is used to remove only (small) higher-order corrections in the spectral density. The details of these fits are reported in the next two subsections.

\section{A. Continuum limit fit}

In the first strategy outlined in Sec. IV, we start by interpolating the data in the quark mass at fixed $\Lambda_{R}$ and $a$. We choose three reference values $\left(m_{R}=12.9,20.9\right.$, 32.0 MeV) which are within the range of simulated quark masses at all $\beta$ values, and they are as close as possible to the values at the finest lattice spacing. Most of the data sets look perfectly linear in $m$ in the vicinity of the interpolation points, with small deviations only for simultaneous coarse lattices, light $\Lambda_{R}$ 's and towards heavy quark masses (see Fig. 4). In all cases, however, the systematic error associated with the linear interpolation is negligible with respect to the statistical one. The interpolation and all following fits are performed using the jackknife technique to take into account the correlation of the data.

At fixed $\left(\Lambda_{R}, m_{R}\right)$, each data set is well fitted by a linear function in $a^{2}$, see Fig. 2, a fact which supports the assumption of being in the Symanzik asymptotic regime within the errors quoted. ${ }^{6}$ Once extrapolated to the continuum limit, we fit the effective spectral density with the functional form

$$
\begin{aligned}
\tilde{\rho}_{R}= & c_{0}\left(\Lambda_{R}\right)+m_{R}\left[c_{1}+c_{2}\left(-3 \ln \left(\frac{m_{R}}{\bar{\mu}}\right)\right.\right. \\
& \left.\left.+\tilde{g}_{\nu}\left(\frac{\Lambda_{R, 1}}{m_{R}}, \frac{\Lambda_{R, 2}}{m_{R}}\right)\right)\right],
\end{aligned}
$$

which rests on NLO ChPT but is capable of accounting for $\mathrm{O}\left(\Lambda^{2}\right)$ effects. The latter are expected to be the dominant higher-order effects in ChPT in this range of parameters. Within the given accuracy, $c_{0}(\Lambda)$ is consistent with a plateau behavior in the range $20 \leq \Lambda_{R} \leq 80 \mathrm{MeV}$; see right plot of Fig. 3. By fitting $c_{0}\left(\Lambda_{R}\right)$ to a constant in this range, we obtain $\Sigma^{1 / 3}=261(6) \mathrm{MeV}$. If we include also a $\Lambda_{R}^{2}$ term in the fit and consider the entire range $20 \leq \Lambda_{R} \leq 120 \mathrm{MeV}$, we find 253(9) $\mathrm{MeV}$, which differs from the previous result by roughly one standard deviation. At the level of our statistical errors of $\mathrm{O}(10 \%)$, the spectral density of the Dirac operator in the continuum and chiral limits is a constant function up to $\Lambda_{R} \approx 80 \mathrm{MeV}$.

\footnotetext{
${ }^{6} \mathrm{~A}$ detailed analysis of discretization effects in the spectral density is beyond the scope of this paper. For completeness, we report the results of these fits in Appendix D for interested readers.
} 


\section{B. Combined fit}

In this section we present an alternative strategy to extract the chiral condensate, based on fitting the data in all three directions $\left(\Lambda_{R}, m_{R}, a\right)$ at the same time. Compared to the first strategy, the shortcomings are that we cannot disentangle different corrections as clearly and ChPT is used from the very beginning. We remark, however, that also in this case ChPT is used only to remove higher-order corrections, while the bulk of the chiral condensate is still given through the Banks-Casher relation. The statistical analysis is based on a double-elimination jackknife fit to take into account all errors and correlations (no fit of fitted quantities is needed). We start with the fit form

$$
\begin{aligned}
\tilde{\rho}_{R}= & c_{0}\left(\Lambda_{R}, a\right)+m_{R}\left[c_{1}\left(\Lambda_{R}, a\right)+c_{2}\left(-3 \ln \left(\frac{m_{R}}{\bar{\mu}}\right)\right.\right. \\
& \left.\left.+\tilde{g}_{\nu}\left(\frac{\Lambda_{R, 1}}{m_{R}}, \frac{\Lambda_{R, 2}}{m_{R}}\right)\right)\right],
\end{aligned}
$$

where $\Lambda_{R}=\left(\Lambda_{R, 1}+\Lambda_{R, 2}\right) / 2$, and we constrain the fit parameters as suggested by NLO chiral and Symanzik effective theories. As already verified in the first strategy, the discretization effects obey an $a^{2}$ dependence in the range of parameters simulated. We, thus, constrain our fit parameters to obey ${ }^{7}$

$$
\begin{aligned}
& c_{0}\left(\Lambda_{R}, a\right)=c_{0,0}\left(\Lambda_{R}\right)+a^{2} c_{0,1}\left(\Lambda_{R}\right), \\
& c_{1}\left(\Lambda_{R}, a\right)=c_{1,0}\left(\Lambda_{R}\right)+a^{2} c_{1,1}\left(\Lambda_{R}\right) .
\end{aligned}
$$

The NLO ChPT predicts that $c_{0,0}\left(\Lambda_{R}\right)$ and $c_{1,0}\left(\Lambda_{R}\right)$ should both be constant. Allowing for the time being an arbitrary $\Lambda_{R}$ dependence in the parameter $c_{0,0}\left(\Lambda_{R}\right)$, we arrive at the fit function

$$
\begin{aligned}
\tilde{\rho}_{R}= & c_{0,0}\left(\Lambda_{R}\right)+a^{2} c_{0,1}\left(\Lambda_{R}\right)+m_{R}\left[c_{1,0}+a^{2} c_{1,1}\left(\Lambda_{R}\right)\right. \\
& \left.+c_{2}\left(-3 \ln \left(\frac{m_{R}}{\bar{\mu}}\right)+\tilde{g}_{\nu}\left(\frac{\Lambda_{R, 1}}{m_{R}}, \frac{\Lambda_{R, 2}}{m_{R}}\right)\right)\right] .
\end{aligned}
$$

The fit of the data is shown versus the quark mass in the left plot of Fig. 4 for the finer lattice spacings and three cutoffs $\Lambda_{R}$ 's. The resulting effective spectral density in the continuum and chiral limit, corresponding to $c_{0,0}\left(\Lambda_{R}\right)$, is shown in the right plot of Fig. 4 . The results are very well compatible with the ones determined in Sec. IV. If we fix $c_{0,0}$ to a constant in the region $20 \leq \Lambda_{R} \leq 80$, we can extract the condensate to get $\Sigma^{1 / 3}=259(6) \mathrm{MeV}$, which is well compatible with the one extracted in the previous strategy.

\footnotetext{
${ }^{7}$ Note that this expression includes also the functional form of discretization effects predicted at NLO in the GSM regime of ChPT [30]; see Appendixes C and E.
}

To assess the stability of the fit, we have amended the function with higher-order terms of the form $\mathcal{O}\left(\Lambda_{R}^{2}, \Lambda_{R} m_{R}, m_{R}^{2}\right)$. Note that when including $\Lambda_{R}^{2}$ terms, we always consider the entire range $20 \leq \Lambda_{R} \leq 120 \mathrm{MeV}$. The coefficient of $\Lambda_{R} m_{R}$ is consistent with zero, while $m_{R}^{2}$ and $\Lambda_{R}^{2}$ effects are nonzero by 2 and 3 standard deviations, respectively, and affect our final result systematically by roughly 1 standard deviation downwards. We remark, however, that in the truncated range $20 \leq \Lambda_{R} \leq 80$ the data is perfectly compatible with a flat dependence on $\Lambda_{R}$. We also investigated the effect of truncating the amount of data included in the fit. Cutting light $\Lambda_{R}$ slightly improves the fit, while cutting heavy ones does not make a noteworthy difference. To check again whether all data obey well the assumed linear $a^{2}$ dependence, we perform also fits excluding the data at the coarsest lattices $(a=0.075 \mathrm{fm})$ with larger discretization effects (we kept 12 out of 32 data points at this lattice spacing). This does not improve the quality of the fit significantly, and it gives $\Sigma^{1 / 3}=267(6) \mathrm{MeV}$ which differs from the previous result by roughly one standard deviation upwards. We remark, however, that the linear $a^{2}$ dependence has been checked and confirmed explicitly for each pair of $\left(\Lambda_{R}, m_{R}\right)$ in the first strategy. A further reduction of the number of fit parameters can actually be achieved by noting that $c_{2}$ is known in ChPT, as seen in Eq. (10). One can rewrite it as a function of $m_{\pi}$ and $m$. We have also tried to fix $c_{0,1}\left(\Lambda_{R}\right)$ to a constant which is suggested from results of the several fits we have done (see Appendix E). In either case, we get results which are well compatible with the results quoted.

For this strategy the best value of the chiral condensate is $\Sigma^{1 / 3}=259(6) \mathrm{MeV}$. It is extracted from the fit function Eq. (14) where $c_{0,0}$ is fitted to a constant in the range $20 \leq \Lambda_{R} \leq 80 \mathrm{MeV}$. This fit confirms that in the chiral and continuum limits the spectral density is a flat function of $\Lambda_{R}$ up to $\approx 80 \mathrm{MeV}$ at the level of precision of our data points in the continuum limit (approximatively 10\%), and it can be parametrized by NLO ChPT.

We presented preliminary results of this study at only two lattice spacings in Ref. [13]. There we observed effects of $\mathcal{O}\left(\Lambda_{R}^{2}\right)$ already for $\Lambda_{R} \gtrsim 50 \mathrm{MeV}$, in particular for $a=0.065 \mathrm{fm}$. Once the data are extrapolated to the continuum limit, these effects are not visible anymore up to $\Lambda_{R} \approx 80 \mathrm{MeV}$. In this respect it must be noted, however, that once the uncertainties in the scale and renormalization constants are included, the final errors of the extrapolated results are significantly larger than those used to study the $\Lambda_{R}$ dependence at fixed lattice spacing. It is, therefore, not surprising that the window extends to larger values of $\Lambda_{R}$.

By estimating the spectral density of the twisted mass Hermitian Dirac operator, the dimensionless quantity $r_{0} \Sigma^{1 / 3}$ was computed in Ref. [31]. Since they have a smaller set of data, the analysis described in Sec. VA is not a viable option for them. They opt for the strategy adopted in Ref. [12] which is inspired by NLO ChPT. They fit the 
mode number linearly in $M$ in the range $50-120 \mathrm{MeV}$, and they extrapolate the results to the chiral and continuum limits linearly. The smaller quark masses and in particular the smaller values of $\Lambda_{R}$ that we considered were instrumental to properly quantify and eventually reduce our systematic error.

\section{Finite-size effects}

We have chosen the lattice parameters so that finitevolume effects, as estimated by NLO ChPT (see Appendix C), are negligible within the statistical accuracy. For the lattice E5 we have explicitly checked that finite-size effects are within the expectations of ChPT by comparing the values of the mode number with those obtained on a lattice of $48 \times 24^{3}$, lattice D5 in Table VI of Appendix D.

\section{RESULTS AND CONCLUSIONS}

Our results show that in QCD with two flavors, the low modes of the Dirac operator do condense in the continuum limit as expected by the Banks-Casher relation in the presence of spontaneous symmetry breaking. The spectral density of the Dirac operator in the chiral limit at the origin is $\left[\pi \rho^{\overline{\mathrm{MS}}}(2 \mathrm{GeV})\right]^{1 / 3}=261(6)(8) \mathrm{MeV}$, where the first error is statistical and the second is systematic. The latter is estimated so that the results from various fits are within the range covered by the systematic error: in particular, the smaller value that we find in Sec. VA when a $\Lambda_{R}^{2}$ term is included in the fit function and the higher one obtained in Sec. V B when some of the data at the coarser lattice spacing are excluded from the fit. From the GMOR relation, the best value of the chiral condensate that we obtain is $\left[\Sigma_{\mathrm{GMOR}}^{\overline{\mathrm{MS}}}(2 \mathrm{GeV})\right]^{1 / 3}=263(3)(4) \mathrm{MeV}$, where again the first error is statistical and the second is systematic. The spectral density at the origin, thus, agrees with $M_{\pi}^{2} F_{\pi}^{2} /\left(2 m_{R}\right)$ when both are extrapolated to the chiral limit.

For the sake of clarity, the above values of the condensate have been expressed in physical units by supplementing the theory with a quenched "strange" quark and by fixing the lattice spacing from the kaon decay constant $F_{K}$. They are, therefore, affected by an intrinsic ambiguity due to the matching of $F_{K}$ in the $N_{f}=2$ partially quenched theory with its experimental value. The renormalization groupinvariant dimensionless ratio

$$
\frac{\left[\Sigma^{\mathrm{RGI}}\right]^{1 / 3}}{F}=2.77(2)(4),
$$

however, is a parameter-free prediction of the $N_{f}=2$ theory. It belongs to the family of unambiguous quantities that should be used for comparing computations in the twoflavor theory rather than those expressed in physical units [6].

\section{ACKNOWLEDGMENTS}

Simulations have been performed on BlueGene/Q at CINECA (CINECA-INFN agreement), on HLRN, on JUROPA/JUQUEEN at Jülich JSC, on PAX at DESYZeuthen, and on Wilson at Milano-Bicocca. We thank these institutions for the computer resources and the technical support. We are grateful to our colleagues within the CLS initiative for sharing the ensembles of gauge configurations. G. P. E. and L. G. acknowledge partial support by the MIURPRIN Contract No. 20093BMNNPR and by the INFN SUMA project. S. L. and R. S. acknowledge support by the DFG Sonderforschungsbereich/Transregio SFB/TR9.

\section{APPENDIX A: LATTICE ACTION AND OPERATORS}

The gluons are discretized with the Wilson plaquette action, while the doublet of mass-degenerate quarks with the $\mathrm{O}(a)$-improved Wilson action ${ }^{8}[17,18]$ with its coefficient $c_{\mathrm{sw}}$ determined nonperturbatively [32]. We are interested in the flavor nonsinglet $(r, s=1,2 ; r \neq s)$ fermion bilinears

$$
P^{r s}=\bar{\psi}_{r} \gamma_{5} \psi_{s}, \quad A_{0}^{r s}=\bar{\psi}_{r} \gamma_{0} \gamma_{5} \psi_{s} .
$$

The corresponding $\mathrm{O}(a)$-improved renormalized operators are given by

$$
\begin{aligned}
P_{R}^{r s} & =Z_{\mathrm{P}}\left(1+\left(\bar{b}_{\mathrm{P}}+\tilde{b}_{\mathrm{P}}\right) a m\right) P^{r s}, \\
A_{0, R}^{r s} & =Z_{\mathrm{A}}\left(1+\left(\bar{b}_{\mathrm{A}}+\tilde{b}_{\mathrm{A}}\right) a m\right)\left\{A_{0}^{r s}+c_{\mathrm{A}} \frac{a}{2}\left(\partial_{0}^{*}+\partial_{0}\right) P^{r s}\right\},
\end{aligned}
$$

where $\partial_{0}$ and $\partial_{0}^{*}$ are the forward and the backward lattice derivatives, respectively. The coefficient $c_{\mathrm{A}}$ has been determined nonperturbatively for the $N_{f}=2$ theory in Ref. [33], while the $b$ coefficients are known in perturbation theory up to one loop only $[34,35]$. The multiplicative renormalization constants $Z_{\mathrm{A}}$ and $Z_{\mathrm{P}}$ have been computed nonperturbatively in Ref. [24]. For the lattices considered in this paper, the numerical values of the improvement coefficients and of the renormalization constants are summarized in Table III. The matching factors between $Z_{\mathrm{P}}$ in the Schrödinger functional scheme and the renormalization-group invariant $Z_{\mathrm{P}}^{\mathrm{RGI}}$ (with the overall normalization convention of Ref. [24]) and $Z_{\mathrm{P}}^{\overline{\mathrm{MS}}}(2 \mathrm{GeV})$ are

$$
\begin{aligned}
Z_{\mathrm{P}}^{\mathrm{RGI}} & =\frac{1}{1.308(16)} Z_{\mathrm{P}}, \\
Z_{\mathrm{P}}^{\overline{\mathrm{MS}}}(2 \mathrm{GeV}) & =\frac{1}{0.740(12)} Z_{\mathrm{P}}^{\mathrm{RGI}} .
\end{aligned}
$$

\footnotetext{
${ }^{8}$ The correction proportional to $b_{\mathrm{g}}$ is neglected.
} 
TABLE III. Improvement coefficients and renormalization constants for the $\beta$ values considered in the paper.

\begin{tabular}{lcccccccc}
\hline \hline$\beta$ & run & $c_{\mathrm{SW}}$ & $c_{\mathrm{A}}$ & $\tilde{b}_{\mathrm{P}}$ & $\tilde{b}_{\mathrm{A}}$ & $\bar{b}_{\mu}$ & $Z_{\mathrm{P}}$ & $Z_{\mathrm{A}}$ \\
\hline 5.2 & all & 2.01715 & -0.06414 & 1.07224 & 1.07116 & -0.576 & $0.5184(53)$ & $0.7703(57)$ \\
5.3 & all & 1.90952 & -0.05061 & 1.07088 & 1.06982 & -0.575 & $0.5184(53)$ & $0.7784(52)$ \\
5.5 & $\mathrm{~N} 5$ & 1.751496 & -0.03613 & 1.06830 & 1.06728 & -0.572 & $0.5184(53)$ & $0.7932(43)$ \\
5.5 & $\mathrm{~N} 6, \mathrm{O} 7$ & 1.751500 & -0.03613 & 1.06830 & 1.06728 & -0.572 & $0.5184(53)$ & $0.7932(43)$ \\
\hline \hline
\end{tabular}

Using the PCAC relation, we can define

$$
m\left(x_{0}\right)=\frac{\frac{1}{2}\left(\partial_{0}+\partial_{0}^{*}\right) f_{\mathrm{AP}}\left(x_{0}\right)+c_{\mathrm{A}} a \partial_{0}^{*} \partial_{0} f_{\mathrm{PP}}\left(x_{0}\right)}{2 f_{\mathrm{PP}}\left(x_{0}\right)},
$$

where

$$
\begin{aligned}
& f_{\mathrm{PP}}\left(x_{0}\right)=-a^{3} \sum_{\vec{x}}\left\langle P^{12}(x) P^{21}(0)\right\rangle, \\
& f_{\mathrm{AP}}\left(x_{0}\right)=-a^{3} \sum_{\vec{x}}\left\langle A_{0}^{12}(x) P^{21}(0)\right\rangle .
\end{aligned}
$$

At asymptotically large values of $x_{0}$, the mass $m\left(x_{0}\right)$ has a plateau which defines the value of $m$ to be used in Eqs. (A2). From this, the renormalized quark mass is obtained as

$$
m_{R}=\frac{Z_{\mathrm{A}}\left(1+\left(\bar{b}_{\mathrm{A}}+\tilde{b}_{\mathrm{A}}\right) a m\right)}{Z_{\mathrm{P}}\left(1+\left(\bar{b}_{\mathrm{P}}+\tilde{b}_{\mathrm{P}}\right) a m\right)} m
$$

where the renormalization scheme and scale are those of $Z_{\mathrm{P}}$. In particular in this paper, when not explicitly stated, we use the symbol $m_{R}$ for the quark mass renormalized in the $\overline{\mathrm{MS}}$ scheme at $\mu=2 \mathrm{GeV}$. The bare pseudoscalar decay constant is given by [36]

$$
\mathcal{F}_{\pi}=2 m \frac{G_{\pi}}{M_{\pi}^{2}},
$$

where $G_{\pi}$ is extracted from the behavior of the correlator $f_{\mathrm{PP}}\left(x_{0}\right)$ at asymptotically large values of $x_{0}$,

$$
f_{\mathrm{PP}}\left(x_{0}\right)=\frac{G_{\pi}^{2}}{M_{\pi}} e^{-M_{\pi} x_{0}} .
$$

Thanks to Eq. (A2), the pseudoscalar decay constant is finally given by

$$
F_{\pi}=Z_{\mathrm{A}}\left(1+\left(\bar{b}_{\mathrm{A}}+\tilde{b}_{\mathrm{A}}\right) a m\right) \mathcal{F}_{\pi} .
$$

\section{APPENDIX B: QUARK MASSES, PION MASSES AND DECAY CONSTANTS}

On all ensembles in Table I, we have computed the twopoint functions of the flavor nonsinglet bilinear operators in Eqs. (A4) and (A5). They have been estimated by using
10 to $20 U(1)$ noise sources located on randomly chosen time slices. The bare quark mass $m\left(x_{0}\right)$ in Eq. (A4) has a plateau for large enough $x_{0}$ over which we average. The pion mass $M_{\pi}$ and the bare pion decay constant $\mathcal{F}_{\pi}$ are extracted from $f_{\mathrm{PP}}\left(x_{0}\right)$ and the quark mass following Ref. [24]. In particular, we determine the region $x_{0} \in$ $\left[x_{0}^{\min } ; T-x_{0}^{\min }\right]$ where we can neglect the excited state contribution by first fitting the pseudoscalar two-point function with a two-exponential fit,

$$
\begin{aligned}
f_{\mathrm{PP}}\left(x_{0}\right)= & d_{1}\left[e^{-E_{1} x_{0}}+e^{-E_{1}\left(T-x_{0}\right)}\right] \\
& +d_{2}\left[e^{-E_{2} x_{0}}+e^{-E_{2}\left(T-x_{0}\right)}\right],
\end{aligned}
$$

in a range where this function describes the data well for the given statistical accuracy. We then determine $x_{0}^{\min }$ to be the smallest value of $x_{0}$ where the statistical uncertainty on the effective mass $m_{\text {eff }}\left(x_{0}\right)=-\frac{\mathrm{d}}{\mathrm{d} x_{0}} \log \left[f_{\mathrm{PP}}\left(x_{0}\right)\right]$ is 4 times larger than the contribution of the excited state to $m_{\text {eff }}\left(x_{0}\right)$ as given by the result of the fit. In the second step only the first term of Eq. (B1) is fitted to the data restricted to this region, and $E_{1}$ and $d_{1}$ are determined. The pion mass and its decay constant are then fixed to be $M_{\pi}=E_{1}$ and $\mathcal{F}_{\pi}=2 \sqrt{d_{1}} m / M_{\pi}^{3 / 2}$, respectively. The numerical results for all lattices are reported in Table IV, and those for the pseudoscalar decay constant and for the cubic root of the ratio $M_{\pi}^{2} /\left(2 m_{R} F\right)$ are shown in Fig. 5 versus $y=M_{\pi}^{2} /\left(4 \pi F_{\pi}\right)^{2}$. We fit $F_{\pi}$ to the function

TABLE IV. The bare quark mass am as defined in Eq. (A4), the pion mass $a M_{\pi}$ and pion decay constant $a F_{\pi}$ as defined in Eq. (A9).

\begin{tabular}{lllc}
\hline \hline id & \multicolumn{1}{c}{$a m$} & \multicolumn{1}{c}{$a M_{\pi}$} & $a F_{\pi}$ \\
\hline A3 & $0.00985(6)$ & $0.1883(8)$ & $0.04583(37)$ \\
A4 & $0.00601(6)$ & $0.1466(8)$ & $0.04200(35)$ \\
A5 & $0.00444(6)$ & $0.1263(11)$ & $0.04023(34)$ \\
B6 & $0.00321(4)$ & $0.1073(8)$ & $0.03883(31)$ \\
E5 & $0.00727(3)$ & $0.1454(5)$ & $0.03803(29)$ \\
F6 & $0.00374(3)$ & $0.1036(5)$ & $0.03479(29)$ \\
F7 & $0.002721(20)$ & $0.0886(4)$ & $0.03331(24)$ \\
G8 & $0.001395(18)$ & $0.0638(4)$ & $0.03162(23)$ \\
N5 & $0.00576(3)$ & $0.1085(8)$ & $0.02816(21)$ \\
N6 & $0.003444(15)$ & $0.0837(3)$ & $0.02589(19)$ \\
O7 & $0.002131(9)$ & $0.06574(23)$ & $0.02475(16)$ \\
\hline \hline
\end{tabular}



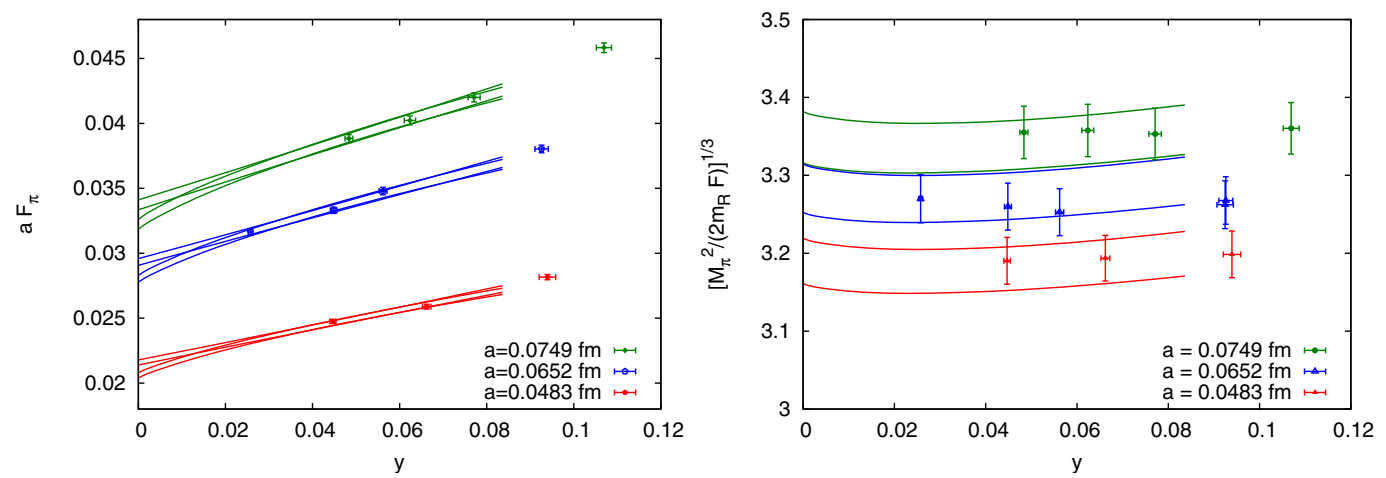

FIG. 5 (color online). Left: the pseudoscalar decay constant $a F_{\pi}$ versus $y=M_{\pi}^{2} /\left(4 \pi F_{\pi}\right)^{2}$. Right: The ratio $M_{\pi}^{2} /\left(2 m_{R} F\right)$ versus $y$. The bands are the result of a combined fit, see main text.

$$
a F_{\pi}=(a F)\{1-y \ln (y)+b y\},
$$

where $b$ is common to all lattice spacings, restricted to the points with $M_{\pi}<400 \mathrm{MeV}$ (see left plot of Fig. 5). This function rests on the Symanzik expansion and is compatible with Wilson ChPT (WChPT) at the NLO [37]. To estimate the systematic error, we performed a number of fits to different functions: linear in $y$ with $M_{\pi}<400 \mathrm{MeV}$ and next-to-next-to-leading order in ChPT with all data included. As a final result, we quote $a F=0.0330(4)(8)$, $0.0287(3)(7)$ and 0.0211(2)(5) at $a=0.075,0.065$ and $0.048 \mathrm{fm}$, respectively, where the second (systematic) error takes into account the spread of the results from the various fits. By fixing the scale from $F_{K}$, and by performing a continuum-limit extrapolation, we obtain our final result $F=85.8(7)(20) \mathrm{MeV}$.

We further compute the ratio $M_{\pi}^{2} /\left(2 m_{R} F\right)$ for all data points. We fit the data restricted to $M_{\pi}<400 \mathrm{MeV}$ to

$\left[\frac{M_{\pi}^{2}}{2 m_{R} F}\right]^{1 / 3}=\left(s_{0}+s_{1}(a F)^{2}\right)\left\{1+\frac{y}{6} \ln (y)+d y\right\}$,

where $s_{0}, s_{1}$ and $d$ are common to all lattice spacings, and the fit function is again the one resting on the Symanzik expansion and compatible with WChPT at the NLO. Also in this case we checked several variants, although the data look very flat up to the heaviest mass. From the fits we get $s_{0}=3.06(3)(4)$, where the systematic error is determined as for $F$. This translates to a value for the renormalization-group-invariant dimensionless ratio of $\left[\Sigma^{\mathrm{RGI}}\right]^{1 / 3} / F=2.77(2)(4)$, which in turn corresponds to $\left[\Sigma^{\overline{\mathrm{MS}}}(2 \mathrm{GeV})\right]^{1 / 3}=263(3)(4) \mathrm{MeV}$ if again $F_{K}$ is used to set the scale.

\section{APPENDIX C: MODE NUMBER IN CHIRAL PERTURBATION THEORY}

When chiral symmetry is spontaneously broken, the mode number can be computed in the chiral effective theory. At the NLO it reads [12] (see also Ref. [38])

$$
\begin{aligned}
\nu^{\mathrm{nlo}}\left(\Lambda_{R}, m_{R}\right)= & \frac{2 \Sigma \Lambda_{R} V}{\pi}\left\{1+\frac{m_{R} \Sigma}{(4 \pi)^{2} F^{4}}\left[3 \bar{l}_{6}+1-\ln (2)\right.\right. \\
& \left.\left.-3 \ln \left(\frac{\Sigma m_{R}}{F^{2} \bar{\mu}^{2}}\right)+f_{\nu}\left(\frac{\Lambda_{R}}{m_{R}}\right)\right]\right\}
\end{aligned}
$$

where

$$
\begin{aligned}
f_{\nu}(x)= & x\left[\arctan (x)-\frac{\pi}{2}\right]-\frac{1}{x} \arctan (x) \\
& -\ln (x)-\ln \left(1+x^{2}\right)
\end{aligned}
$$

The constants $F$ and $\bar{l}_{6}$ are, respectively, the pion decay constant in the chiral limit and a $\mathrm{SU}(3 \mid 1)$ low-energy effective coupling renormalized at the scale $\bar{\mu}$. The formula in Eq. (C1) has some interesting properties:

(i) for $x \rightarrow \infty$,

$$
f_{\nu}(x) \underset{x \rightarrow \infty}{\longrightarrow}-3 \ln (x)
$$

and, therefore, at fixed $\Lambda_{R}$ the mode number has no chiral logs when $m_{R} \rightarrow 0$;

(ii) since in the continuum the operator $D_{m}^{\dagger} D_{m}$ has a threshold at $\alpha=m^{2}$, the mode number must satisfy

$$
\lim _{\Lambda_{R} \rightarrow 0} \nu^{\text {nlo }}\left(\Lambda_{R}, m_{R}\right)=0,
$$

a property which is inherited by the NLO ChPT formula;

(iii) in the chiral limit $\nu^{\text {nlo }}\left(\Lambda_{R}, m_{R}\right) / \Lambda_{R}$ becomes independent of $\Lambda_{R}$. This is an accident of the $N_{f}=2$ ChPT theory at NLO [29];

(iv) the $\Lambda_{R}$ dependence in the square brackets on the rhs of $(\mathrm{C} 1)$ is parameter free. Since $\frac{m_{R} \Sigma^{2}}{(4 \pi)^{2} F^{4}}>0$, the behavior of the function $f_{\nu}(x)$ implies that $\nu^{\text {nlo }}\left(\Lambda_{R}, m_{R}\right) / \Lambda_{R}$ is a decreasing function of $\Lambda_{R}$ at fixed $m_{R}$, and no ambiguity is left due to free parameters. 
At the NLO the effective spectral density defined in Eq. (5) reads

$$
\begin{aligned}
\tilde{\rho}_{R}^{\text {nlo }}= & \Sigma\left\{1+\frac{m_{R} \Sigma}{(4 \pi)^{2} F^{4}}\left[3 \bar{l}_{6}+1-\ln (2)\right.\right. \\
& \left.\left.-3 \ln \left(\frac{\Sigma m_{R}}{F^{2} \bar{\mu}^{2}}\right)+\tilde{g}_{\nu}\left(\frac{\Lambda_{1, R}}{m_{R}}, \frac{\Lambda_{2, R}}{m_{R}}\right)\right]\right\},
\end{aligned}
$$

where

$\tilde{g}_{\nu}\left(x_{1}, x_{2}\right)=\frac{f_{\nu}\left(x_{1}\right)+f_{\nu}\left(x_{2}\right)}{2}+\frac{1}{2} \frac{x_{1}+x_{2}}{x_{2}-x_{1}}\left[f_{\nu}\left(x_{2}\right)-f_{\nu}\left(x_{1}\right)\right]$.

The quantity $\tilde{\rho}_{R}^{\text {nlo }}$ inherits the same peculiar properties of $\nu^{\text {nlo }}\left(\Lambda_{R}, m_{R}\right) / \Lambda_{R}$ at NLO: at fixed $\Lambda_{1, R}$ and $\Lambda_{2, R}$, it has no chiral logarithms when $m_{R} \rightarrow 0$, it is independent from $\Lambda_{1, R}$ and $\Lambda_{2, R}$ in the chiral limit, and at nonzero quark mass it is a decreasing parameter-free (apart the overall factor) function of $\left(\Lambda_{1, R}+\Lambda_{2, R}\right) / 2$. It is very weakly dependent on $\left(\Lambda_{1, R}-\Lambda_{2, R}\right)$ in the range we are interested in. To have a quantitative idea of the $\left(\Lambda_{1, R}+\Lambda_{2, R}\right) / 2$ dependence of $\tilde{\rho}_{R}^{\text {nlo }}$, we can choose $\Sigma=(260 \mathrm{MeV})^{3}, F=85 \mathrm{MeV}$,

$$
\begin{aligned}
\left(\frac{\Delta \nu_{V}}{\nu}\right)^{\text {nlo }}= & \frac{\Sigma}{(4 \pi)^{2} F^{4}} \sum_{\left\{n_{1}, \ldots, n_{4}\right\}}^{\prime} \lim _{\epsilon \rightarrow 0}\left\{\frac{2}{\Lambda_{R}} \operatorname{Im}\left[F_{-2}\left(\frac{\Sigma q_{n}^{2}}{4 F^{2}}, i \Lambda_{R}+m_{R}+\epsilon\right)\right]-\frac{m_{R}}{\Lambda_{R}} \operatorname{Im}\left[F_{-1}\left(\frac{\Sigma q_{n}^{2}}{2 F^{2}}, i \Lambda_{R}+\epsilon\right)\right]\right. \\
& \left.+\operatorname{Re}\left[F_{-1}\left(\frac{\Sigma q_{n}^{2}}{2 F^{2}}, i \Lambda_{R}+\epsilon\right)\right]\right\},
\end{aligned}
$$

where

$$
F_{\nu}(b, z)=2\left(\frac{b}{z}\right)^{\nu / 2} K_{\nu}(2 \sqrt{b z})
$$

with $\operatorname{Re} b>0, \operatorname{Re} z>0$, and $K_{\nu}$ is a modified Bessel function [39]. Furthermore, $q_{n}^{2}=\sum_{\mu=1}^{d}\left(n_{\mu} L_{\mu}\right)^{2}$ and $\sum_{\left\{n_{1}, \ldots, n_{d}\right\}}^{\prime}$ denotes the sum over all integers without $n=(0, \ldots, 0)$. By expanding the Bessel functions for large arguments [39], it is straightforward to show that the most significant terms in the sum on the rhs of Eq. (C9) are proportional to the exponentials $\exp \left\{-M_{1} L / \sqrt{2}\right\}$ and $\exp \left\{-M_{2} L / 2\right\}$, where $M_{1}$ and $M_{2}$ are the leading-order expressions in ChPT for the mass of a pseudoscalar meson made of two valence quarks of mass $\Lambda_{R}$ and $\left(\sqrt{\Lambda_{R}^{2}+m_{R}^{2}}+m_{R}\right)$, respectively.

\section{Discretization effects}

At finite lattice spacing and volume, the threshold region should be treated carefully in ChPT [19]. The latter
$m_{R}^{\text {sea }}=10 \mathrm{MeV}, \Lambda_{1, R}=20,40 \mathrm{MeV}, \Lambda_{2, R}=25,55 \mathrm{MeV}$ to obtain

$0.0213 \cdot\left[\tilde{g}_{\nu}\left(\frac{20}{10}, \frac{25}{10}\right)-\tilde{g}_{\nu}\left(\frac{40}{10}, \frac{55}{10}\right)\right]=0.0467$.

For light values of the quark masses, the variations are rather mild, i.e. of the order of a few percent. The next-tonext-to-leading corrections in $\tilde{\rho}_{R}$ are of the form $\mathcal{O}\left(\Lambda_{R}^{2}, m_{R} \Lambda_{R}, m_{R}^{2}\right)$. They are expected to spoil some of the peculiar properties of the NLO formula. In the chiral limit the $\mathcal{O}\left(\Lambda_{R}^{2}\right)$ corrections can induce a $\Lambda_{R}$ dependence, and the $\mathcal{O}\left(m_{R} \Lambda_{R}\right)$ can change the parameter-free dependence on $\Lambda_{R}$ within the square brackets on the rhs of Eq. (C5).

\section{Finite volume effects}

Finite volume effects in the mode number were computed in the chiral effective theory at the NLO in Refs. [12,38] (see also [30]). They are given by can be avoided by considering the quantity $\tilde{\rho}_{R}$, with $\Lambda_{2, R}>\Lambda_{1, R} \gg 1 / \Sigma V$. In this case, the computation in the GSM power-counting regime of the Wilson ChPT gives [30]

$$
\tilde{\rho}_{R}^{\mathrm{nlo}}(a)=\tilde{\rho}_{R}^{\mathrm{nlo}}-32\left(W_{0} a\right)^{2} W_{8}^{\prime} m_{R} \frac{1}{\Lambda_{1, R} \Lambda_{2, R}} .
$$

Since $W_{8}^{\prime}$ is expected to be negative $[20,40]$, if we rewrite

$$
\Lambda_{1, R} \Lambda_{2, R}=\left(\frac{\Lambda_{1, R}+\Lambda_{2, R}}{2}\right)^{2}-\frac{1}{4}\left(\Lambda_{2, R}-\Lambda_{1, R}\right)^{2},
$$

and we keep constant $\left(\Lambda_{2, R}-\Lambda_{1, R}\right)$, then $\tilde{\rho}_{R}^{\text {nlo }}(a)$ is a decreasing function of $\Lambda_{R}=\left(\Lambda_{2, R}+\Lambda_{1, R}\right) / 2$ on the lattice too. At variance with the continuum case, however, a free parameter $W_{0}^{2} W_{8}^{\prime}$ appears in the function, and its magnitude cannot be predicted. Remarkably, $\tilde{\rho}_{R}^{\text {nlo }}(a)$ is free from discretization effects in the chiral limit and, 
therefore, it is independent of $\Lambda_{1, R}$ and $\Lambda_{2, R}$. The continuum extrapolation of the chiral value of $\tilde{\rho}_{R}^{\text {nlo }}(a)$ then removes the discretization effects due to the reference scale used.

\section{APPENDIX D: NUMERICAL RESULTS FOR THE MODE NUMBER}

We collect the results for the mode number in Tables V, VI and VII. For each lattice, the values of $a M$ correspond to approximatively $\Lambda_{R}=20,25,30,40,55,71$, $86,101,116 \mathrm{MeV}$ with the exception of the lattice E5 for which $\Lambda_{R}=151,202,303,505 \mathrm{MeV}$ were also computed. In Table VIII we give the value of $\tilde{\rho}_{\mathrm{R}}$ in the

TABLE V. Values of $a M$ and the corresponding results for $\nu$ for each lattice at $\beta=5.2$.

\begin{tabular}{|c|c|c|c|c|c|c|c|}
\hline \multirow{3}{*}{\multicolumn{4}{|c|}{$\begin{array}{l}\text { tinuum extrapolation of the chiral value of } \tilde{\rho}_{R}^{\text {nlo }}(a) \text { then } \\
\text { removes the discretization effects due to the reference } \\
\text { scale used. }\end{array}$}} & \multirow{3}{*}{$\frac{\text { id }}{\text { D5 }}$} & \multirow{2}{*}{$\frac{N_{\text {cnfgs }}}{345}$} & $a M$ & $\nu$ \\
\hline & & & & & & 0.006720 & $2.09(9)$ \\
\hline & & & & & & 0.007239 & $2.77(10)$ \\
\hline \multirow{3}{*}{\multicolumn{4}{|c|}{$\begin{array}{l}\text { APPENDIX D: NUMERICAL RESULTS } \\
\text { FOR THE MODE NUMBER }\end{array}$}} & & & 0.007826 & $3.42(10)$ \\
\hline & & & & & & 0.009153 & $5.26(12)$ \\
\hline & & & & & & 0.011385 & $8.38(16)$ \\
\hline \multirow{6}{*}{\multicolumn{4}{|c|}{$\begin{array}{l}\text { We collect the results for the mode number in } \\
\text { Tables V, VI and VII. For each lattice, the values of } a M \\
\text { correspond to approximatively } \Lambda_{R}=20,25,30,40,55,71 \text {, } \\
86,101,116 \mathrm{MeV} \text { with the exception of the lattice } \\
\text { E5 for which } \Lambda_{R}=151,202,303,505 \mathrm{MeV} \text { were also } \\
\text { computed. In Table VIII we give the value of } \tilde{\rho}_{\mathrm{R}} \text { in the }\end{array}$}} & & & 0.013782 & $11.69(19)$ \\
\hline & & & & & & 0.016271 & $15.16(22)$ \\
\hline & & & & & & 0.018815 & $18.61(25)$ \\
\hline & & & & & & 0.021396 & $22.3(3)$ \\
\hline & & & & E5 & 92 & 0.006720 & 7.3(3) \\
\hline & & & & & & 0.007239 & $9.3(3)$ \\
\hline & & & & & & 0.007826 & $11.5(3)$ \\
\hline & & & & & & 0.009153 & 17.1(4) \\
\hline \multirow{2}{*}{\multicolumn{4}{|c|}{$\begin{array}{l}\text { TABLE V. Values of } a M \text { and the corresponding results for } \nu \text { for } \\
\text { each lattice at } \beta=5.2 \text {. }\end{array}$}} & & & 0.011385 & 26.9(5) \\
\hline & & & & & & 0.013782 & $37.4(7)$ \\
\hline id & $N_{\text {cnfgs }}$ & $a M$ & $\nu$ & & & 0.016271 & 47.3(8) \\
\hline \multirow{9}{*}{ A3 } & & & & & & 0.018815 & $58.0(9)$ \\
\hline & 55 & 0.008673 & 13.3(6) & & & 0.021396 & $68.8(10)$ \\
\hline & & 0.009208 & $16.2(6)$ & & & 0.027499 & $93.7(10)$ \\
\hline & & 0.009821 & $20.5(7)$ & & & 0.036321 & $138.6(12)$ \\
\hline & & 0.011235 & $\begin{array}{l}29.6(9) \\
473(10)\end{array}$ & & & 0.054110 & $259.7(16)$ \\
\hline & & $\begin{array}{l}0.013665 \\
0.016322\end{array}$ & $\begin{array}{l}47.3(10) \\
66.9(12)\end{array}$ & & & 0.089863 & 689(3) \\
\hline & & 0.019110 & $88.2(14)$ & F6 & 50 & 0.004618 & $34.7(9)$ \\
\hline & & 0.021979 & 111.1(16) & & & 0.005342 & $47.6(11)$ \\
\hline & & 0.024901 & $134.6(18)$ & & & 0.006111 & $60.7(12)$ \\
\hline \multirow[t]{8}{*}{ A } & 55 & 0.006205 & 11.6(6) & & & 0.007732 & $90.8(16)$ \\
\hline & & 0.006929 & $15.9(7)$ & & & 0.010268 & $135.8(17)$ \\
\hline & & 0.007723 & 20.6(7) & & & 0.012865 & $183.0(20)$ \\
\hline & & 0.009447 & $30.8(8)$ & & & 0.015492 & $230.9(23)$ \\
\hline & & $\begin{array}{l}0.012228 \\
0.015127\end{array}$ & $\begin{array}{l}48.8(10) \\
68.6(12)\end{array}$ & & & 0.018137 & $280(3)$ \\
\hline & & 0.018088 & $89.6(13)$ & & & 0.020791 & $330(3)$ \\
\hline & & 0.021085 & $110.9(15)$ & F7 & 50 & 0.004159 & $34.7(9)$ \\
\hline & & 0.024103 & $132.5(15)$ & & & 0.004950 & $47.0(10)$ \\
\hline \multirow[t]{8}{*}{ A5 } & 55 & 0.005352 & 11.4(6) & & & 0.005770 & $59.3(10)$ \\
\hline & & 0.006176 & $15.6(6)$ & & & 0.007464 & $87.1(12)$ \\
\hline & & 0.007054 & $20.6(7)$ & & & 0.010065 & $128.9(16)$ \\
\hline & & 0.008905 & $31.9(8)$ & & & 0.012701 & $172.0(21)$ \\
\hline & & 0.011810 & $50.1(11)$ & & & 0.015354 & $217.2(23)$ \\
\hline & & $\begin{array}{l}0.014786 \\
0.017799\end{array}$ & $\begin{array}{l}68.3(13) \\
88.7(14)\end{array}$ & & & 0.018015 & $265(3)$ \\
\hline & & 0.020831 & $108.7(16)$ & & & 0.020682 & $314(3)$ \\
\hline & & 0.023877 & $129.2(18)$ & G8 & 50 & 0.003737 & $113.7(16)$ \\
\hline \multirow[t]{8}{*}{ B6 } & 50 & 0.004800 & $59.5(10)$ & & & 0.004599 & $153.8(18)$ \\
\hline & & 0.005703 & $82.5(11)$ & & & 0.005472 & $196.7(22)$ \\
\hline & & 0.006642 & $108.4(13)$ & & & 0.007233 & $282.3(25)$ \\
\hline & & 0.008580 & $162.3(16)$ & & & 0.009892 & 409(3) \\
\hline & & 0.011563 & 253.0(22) & & & 0.012560 & $543(3)$ \\
\hline & & $\begin{array}{l}0.014586 \\
0.017629\end{array}$ & $\begin{array}{c}346.5(25) \\
443(3)\end{array}$ & & & 0.015233 & $682(4)$ \\
\hline & & $\begin{array}{l}0.01 / 229 \\
0.020683\end{array}$ & $\begin{array}{l}443(3) \\
543(3)\end{array}$ & & & 0.017910 & $828(4)$ \\
\hline & & 0.023743 & $647(4)$ & & & 0.020587 & $981(5)$ \\
\hline
\end{tabular}

TABLE VI. As in Table V but for $\beta=5.3$. 
TABLE VII. As in Table V but for $\beta=5.5$.

\begin{tabular}{|c|c|c|c|}
\hline id & $N_{\text {cnfgs }}$ & $a M$ & $\nu$ \\
\hline \multirow[t]{9}{*}{ N5 } & 60 & 0.005287 & $12.0(6)$ \\
\hline & & 0.005647 & $15.6(6)$ \\
\hline & & 0.006058 & $19.3(7)$ \\
\hline & & 0.006998 & $27.3(8)$ \\
\hline & & 0.008599 & $40.2(9)$ \\
\hline & & 0.010334 & $52.3(10)$ \\
\hline & & 0.012146 & $65.0(11)$ \\
\hline & & 0.014005 & $77.7(12)$ \\
\hline & & 0.015895 & $91.2(13)$ \\
\hline \multirow[t]{9}{*}{ N6 } & 60 & 0.003797 & $11.0(4)$ \\
\hline & & 0.004284 & $14.9(5)$ \\
\hline & & 0.004812 & $18.3(5)$ \\
\hline & & 0.005949 & $25.6(7)$ \\
\hline & & 0.007765 & $37.3(8)$ \\
\hline & & 0.009646 & 49.1(8) \\
\hline & & 0.011562 & $60.4(9)$ \\
\hline & & 0.013496 & $72.6(10)$ \\
\hline & & 0.015444 & $85.8(11)$ \\
\hline \multirow[t]{9}{*}{ O7 } & 50 & 0.003137 & $34.3(9)$ \\
\hline & & 0.003710 & $45.9(10)$ \\
\hline & & 0.004309 & $57.5(11)$ \\
\hline & & 0.005548 & $78.5(12)$ \\
\hline & & 0.007459 & $111.9(15)$ \\
\hline & & 0.009399 & $147.8(16)$ \\
\hline & & 0.011354 & $184.0(18)$ \\
\hline & & 0.013316 & $220.8(19)$ \\
\hline & & 0.015284 & $260.2(21)$ \\
\hline
\end{tabular}

continuum for various values of the cutoff $\Lambda_{R}$ and the quark mass $m_{\mathrm{R}}$.

\section{APPENDIX E: NUMERICAL ANALYSIS OF DISCRETIZATION EFFECTS}

In this appendix we report more details on the discretization effects that we have observed in our data. We limit

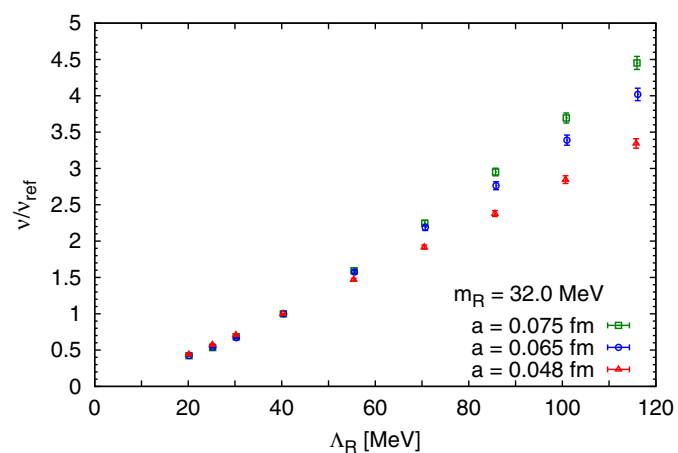

TABLE VIII. The effective density $\tilde{\rho}_{R}$ in the continuum is given for various values of the cutoff $\Lambda_{R}$ and the quark mass $m_{R}$. These data are obtained by first interpolating $\tilde{\rho}_{R}$ linearly in $m_{R}$ for each $\Lambda_{R}$ and lattice spacing $a$, followed by an extrapolation linear in $a^{2}$ to the continuum for each pair of $\left(\Lambda_{R}, m_{R}\right)$, as described in Secs. IV and VA. $\tilde{\rho}_{R}$ is given in $\mathrm{GeV}^{3}$, and $\Lambda_{R}$ and $m_{R}$ are given in $\mathrm{MeV}$.

\begin{tabular}{lcll}
\hline \hline$\Lambda_{R} / m_{R}$ & 12.9 & \multicolumn{1}{c}{20.9} & \multicolumn{1}{c}{32.0} \\
\hline 22.7 & $0.0289(20)$ & $0.032(3)$ & $0.033(3)$ \\
27.7 & $0.0249(21)$ & $0.023(3)$ & $0.029(3)$ \\
35.3 & $0.0191(16)$ & $0.025(3)$ & $0.0308(24)$ \\
47.9 & $0.0192(15)$ & $0.0239(22)$ & $0.0288(19)$ \\
63.0 & $0.0221(15)$ & $0.0228(24)$ & $0.0229(18)$ \\
78.2 & $0.0210(16)$ & $0.0174(20)$ & $0.0224(18)$ \\
93.3 & $0.0212(14)$ & $0.0221(21)$ & $0.0211(18)$ \\
108.4 & $0.0237(15)$ & $0.0257(22)$ & $0.0243(19)$ \\
\hline \hline
\end{tabular}

ourselves to an empirical discussion of the results obtained by following the strategy described in Sec. VA.

A first look into the data reveals that discretization effects in $\nu$ show a nontrivial dependence on $\Lambda_{R}$ and $m_{R}$. We plot the mode number at $m_{R}=32 \mathrm{MeV}$, normalized with respect to its value at $\Lambda_{R}=40 \mathrm{MeV}$, for all three lattice spacings and all values of $\Lambda_{R}$ in Fig. 6, left-hand side. After interpolating the effective spectral density in $m_{R}$, we fit the data linearly in $a^{2}$

$$
\tilde{\rho}_{R}\left(\Lambda_{R}, m_{R}, a\right)=\tilde{\rho}_{R}\left(\Lambda_{R}, m_{R}, 0\right)+a^{2} \Delta\left(\Lambda_{R}, m_{R}\right)
$$

for each pair of $\left(\Lambda_{R}, m_{R}\right)$. By fitting $\Delta$ linearly in $m_{R}$ (Fig. 6, right plot)

$$
\Delta\left(\Lambda_{R}, m_{R}\right)=c_{0,1}\left(\Lambda_{R}\right)+c_{1,1}\left(\Lambda_{R}\right) m_{R}
$$

for each $\Lambda_{R}$, we obtain the values for $c_{0,1}\left(\Lambda_{R}\right)$ shown in the left plot of Fig. 7. Within errors, $c_{0,1}\left(\Lambda_{R}\right)$ turns out to be compatible with a constant. To reduce the noise in $c_{1,1}\left(\Lambda_{R}\right)$,

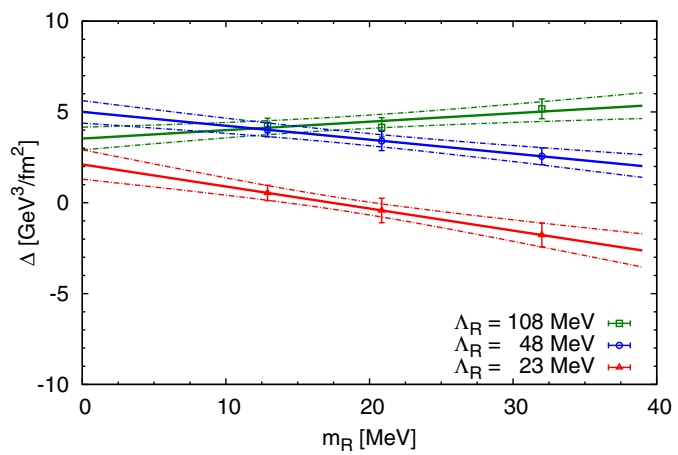

FIG. 6 (color online). Left: mode number at $m_{R}=32 \mathrm{MeV}$ for all three lattice spacings and all cutoffs $\Lambda_{R}$, normalized with respect to its value at $\Lambda_{R}=40 \mathrm{MeV}$. Right: discretization effects $\Delta$ of the effective spectral density as defined in Eq. (E1), shown vs $m_{R}$ for three values of $\Lambda_{R}$. The fit in the plot follows Eq. (E2), the resulting parameters of which are shown in Fig. 7. 

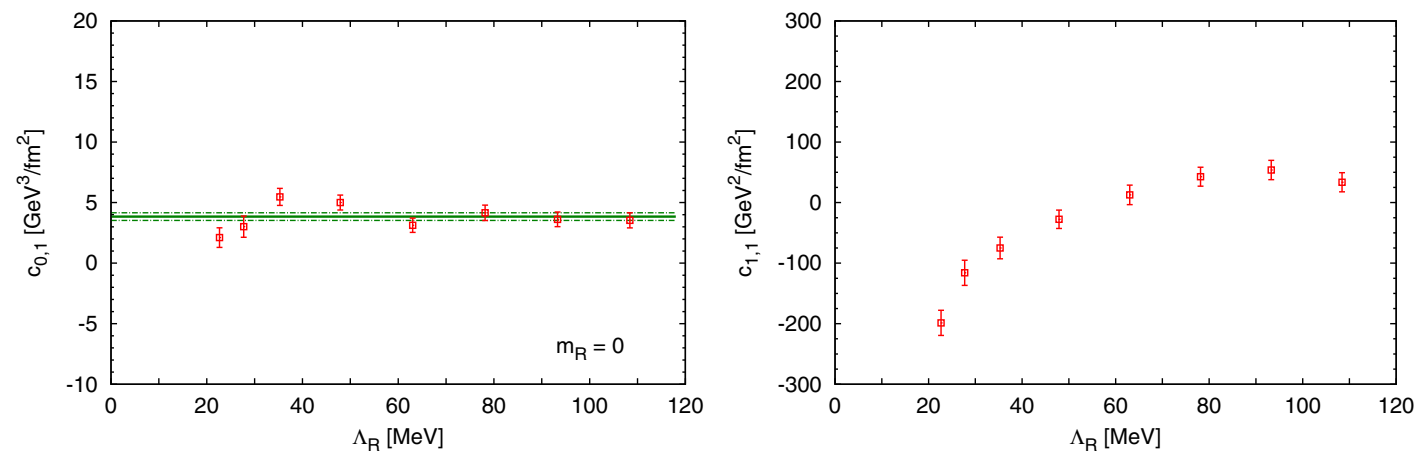

FIG. 7 (color online). Left: mass-independent discretization effects $c_{0,1}$ as defined in Eq. (E2) vs $\Lambda_{R}$. A fit of the plateau gives 3.8(3) $\mathrm{GeV}^{3} / \mathrm{fm}^{2}$. Right: mass-dependent discretization effects $c_{1,1}$ as defined in Eq. (E2) (but with $c_{0,1}\left(\Lambda_{R}\right)$ constrained to be constant), as a function of $\Lambda_{R}$.

we repeat the fit in Eq. (E2) but constraining $c_{0,1}\left(\Lambda_{R}\right)$ to be a constant. The results of this fit are shown in the right plot of Fig. 7. The coefficient $c_{1,1}\left(\Lambda_{R}\right)$ tends to a constant for large $\Lambda_{R}$, while a significant drop is observed towards the origin. In an intermediate range, the opposite signs of $c_{0,1}$ and $c_{1,1}$ allow for a compensation of the different effects, implying an effectively flat dependence of $\tilde{\rho}_{R}$ in the lattice spacing. Within the large errors, the mass-dependent discretization effects could be compatible with the functional form given in Eq. (C10) [30]. The sign of the pole, however, appears to be opposite than predicted in Refs. [20,40]. In this respect it must be said that it is not clear that the GSM power-counting scheme used in Ref. [30] applies in the range of parameters of our data.
[1] M. Hasenbusch, Speeding up the hybrid Monte Carlo algorithm for dynamical fermions, Phys. Lett. B 519, 177 (2001).

[2] M. Lüscher, Schwarz-preconditioned HMC algorithm for two-flavour lattice QCD, Comput. Phys. Commun. 165, 199 (2005).

[3] L. Del Debbio, L. Giusti, M. Lüscher, R. Petronzio, and N. Tantalo, QCD with light Wilson quarks on fine lattices (I): First experiences and physics results, J. High Energy Phys. 02 (2007) 056.

[4] C. Urbach, K. Jansen, A. Shindler, and U. Wenger, HMC algorithm with multiple time scale integration and mass preconditioning, Comput. Phys. Commun. 174, 87 (2006).

[5] M. Lüscher and S. Schaefer, Lattice QCD with open boundary conditions and twisted-mass reweighting, Comput. Phys. Commun. 184, 519 (2013).

[6] S. Aoki, Y. Aoki, C. Bernard, T. Blum, G. Colangelo et al., Review of lattice results concerning low-energy particle physics, Eur. Phys. J. C 74, 2890 (2014).

[7] S. Weinberg, Phenomenological Lagrangians, Physica (Amsterdam) 96A, 327 (1979).

[8] J. Gasser and H. Leutwyler, Chiral perturbation theory to one loop, Ann. Phys. (N.Y.) 158, 142 (1984).

[9] T. Banks and A. Casher, Chiral symmetry breaking in confining theories, Nucl. Phys. B169, 103 (1980).

[10] H. Leutwyler and A. V. Smilga, Spectrum of Dirac operator and role of winding number in QCD, Phys. Rev. D 46, 5607 (1992).
[11] E. V. Shuryak and J. Verbaarschot, Random matrix theory and spectral sum rules for the Dirac operator in QCD, Nucl. Phys. A560, 306 (1993).

[12] L. Giusti and M. Lüscher, Chiral symmetry breaking and the Banks-Casher relation in lattice QCD with Wilson quarks, J. High Energy Phys. 03 (2009) 013.

[13] G. P. Engel, L. Giusti, S. Lottini, and R. Sommer, Chiral condensate from the Banks-Casher relation, Proc. Sci., LATTICE2013 (2014) 119.

[14] G. P. Engel, Chiral condensate in $N_{f}=2$ QCD from the Banks-Casher relation, Proc. Sci., LATTICE2014 (2014) 073.

[15] G. P. Engel, L. Giusti, S. Lottini, and R. Sommer, Chiral Symmetry Breaking in QCD Two Light Flavors, Phys. Rev. Lett. 114, 112001 (2015).

[16] L. Del Debbio, L. Giusti, M. Lüscher, R. Petronzio, and N. Tantalo, Stability of lattice QCD simulations and the thermodynamic limit, J. High Energy Phys. 02 (2006) 011.

[17] B. Sheikholeslami and R. Wohlert, Improved continuum limit lattice action for QCD with Wilson fermions, Nucl. Phys. B259 (1985) 572.

[18] M. Lüscher, S. Sint, R. Sommer, and P. Weisz, Chiral symmetry and $\mathrm{O}(\mathrm{a})$ improvement in lattice QCD, Nucl. Phys. B478, 365 (1996).

[19] P. Damgaard, K. Splittorff, and J. Verbaarschot, Microscopic Spectrum of the Wilson Dirac Operator, Phys. Rev. Lett. 105, 162002 (2010). 
[20] K. Splittorff and J. Verbaarschot, The microscopic twisted mass Dirac spectrum, Phys. Rev. D 85, 105008 (2012).

[21] L. Giusti and S. Necco, Spontaneous chiral symmetry breaking in QCD: A Finite-size scaling study on the lattice, J. High Energy Phys. 04 (2007) 090.

[22] M. Marinkovic and S. Schaefer, Comparison of the mass preconditioned HMC and the DD-HMC algorithm for two-flavour QCD, Proc. Sci., LATTICE2010 (2010) 031.

[23] Lüscher, Martin, DD-HMC algorithm for two-flavour lattice QCD, http://luscher.web.cern.ch/luscher/DD-HMC/ index.html.

[24] P. Fritzsch, F. Knechtli, B. Leder, M. Marinkovic, S. Schaefer, R. Sommer, and F. Virotta, The strange quark mass and Lambda parameter of two flavor QCD, Nucl. Phys. B865, 397 (2012).

[25] P. Fritzsch, P. Korcyl, B. Leder, S. Schaefer, H. Simma, R. Sommer, and F. Virotta (ALPHA Collaboration) (to be published).

[26] M. Marinkovic, S. Schaefer, R. Sommer, and F. Virotta, Strange quark mass and Lambda parameter by the ALPHA Collaboration, Proc. Sci., LATTICE2011 (2011) 232.

[27] M. Bruno, S. Schaefer, and R. Sommer (ALPHA Collaboration), Topological susceptibility and the sampling of field space in $N_{f}=2$ lattice QCD simulations, J. High Energy Phys. 08 (2014) 150.

[28] S. Schaefer, R. Sommer, and F. Virotta (ALPHA Collaboration), Critical slowing down and error analysis in lattice QCD simulations, Nucl. Phys. B845, 93 (2011).

[29] A. V. Smilga and J. Stern, On the spectral density of Euclidean Dirac operator in QCD, Phys. Lett. B 318, 531 (1993).
[30] S. Necco and A. Shindler, Spectral density of the Hermitean Wilson Dirac operator: A NLO computation in chiral perturbation theory, J. High Energy Phys. 04 (2011) 031.

[31] K. Cichy, E. Garcia-Ramos, and K. Jansen, Chiral condensate from the twisted mass Dirac operator spectrum, J. High Energy Phys. 10 (2013) 175.

[32] K. Jansen and R. Sommer (ALPHA Collaboration), O(a) improvement of lattice QCD with two flavors of Wilson quarks, Nucl. Phys. B530, 185 (1998).

[33] M. Della Morte, R. Hoffmann, and R. Sommer, Nonperturbative improvement of the axial current for dynamical Wilson fermions, J. High Energy Phys. 03 (2005) 029.

[34] S. Sint and P. Weisz, Further results on O(a) improved lattice QCD to one loop order of perturbation theory, Nucl. Phys. B502, 251 (1997).

[35] S. Sint and P. Weisz, Further one loop results in $\mathrm{O}(\mathrm{a})$ improved lattice QCD, Nucl. Phys. B, Proc. Suppl. 63, 856 (1998).

[36] L. Del Debbio, L. Giusti, M. Lüscher, R. Petronzio, and N. Tantalo, QCD with light Wilson quarks on fine lattices. II. DD-HMC simulations and data analysis, J. High Energy Phys. 02 (2007) 082.

[37] S. Aoki, O. Bar, and S. R. Sharpe, Vector and axial currents in Wilson chiral perturbation theory, Phys. Rev. D 80, 014506 (2009).

[38] L. Giusti, Spectral density of the QCD Dirac operator at the NLO in chiral perturbation theory, http://virgilio.mib.infn.it/ lgiusti/lgiusti.html, 2008.

[39] M. Abramowitz and I. A. Stegun, Handbook of Mathematical Functions (Dover Publications, New York, 1972).

[40] M. T. Hansen and S. R. Sharpe, Constraint on the low energy constants of Wilson chiral perturbation theory, Phys. Rev. D 85, 014503 (2012). 